\title{
Revista de Estudios y Experiencias en Educación REXE
}

journal homepage: http://revistas.ucsc.cl/index.php/rexe

\section{Consumo de droga en estudiantes ecuatorianos. Una alternativa de prevención y desarrollo resilien- te del alumnado desde la escuela}

\section{Ana Cango Cobos ${ }^{\mathrm{a}}$ y Noemí Suárez Monzón ${ }^{\mathrm{b}}$}

Unidad Educativa CEPE, Puerto Quito ${ }^{a}$. Universidad Tecnológica Indoamérica, Ambato ${ }^{\mathrm{b}}$. Ecuador.

Recibido: 04 de noviembre 2020 - Revisado: 21 de diciembre 2020 - Aceptado: 23 de febrero 2021

\section{RESUMEN}

El consumo de drogas en estudiantes adolescentes ecuatorianos es un fenómeno creciente que se manifiesta a nivel cognitivo, social y en la salud. Los antecedentes lo muestran como un fenómeno multifactorial, ligado a otras adicciones, con mayor presencia en las zonas de extrema pobreza y entornos sociales desfavorecidos. Siendo la escuela uno de los centros que puede contribuir sustancialmente en la toma de decisiones de los adolescentes que conviven en zonas de cultivo, tráfico y consumo de droga, todavía es insuficiente la labor educativa para ganar al avance de este flagelo. La problemática se constató a través de estudio mixto transversal. Se aplicaron 360 encuestas a alumnos entre las edades de 12 a 15 años y una entrevista a la autoridad académica de la institución educativa donde se desarrolló la pesquisa y la funcionaria del Departamento de Consejería Estudiantil. Más del 50\% en algún momento ha consumido drogas y hay evidencias que conducen a la drogodependencia en adolescentes, por consiguiente, al deterioro de su salud. Ante estos hallazgos el objetivo es diagnosticar la situación contextual de la unidad educativa en estudio y ofrecer un sistema de actividades extracurriculares que busca la prevención del consumo de drogas y el desarrollo resiliente del alumnado. Se concluye la viabilidad de la propuesta, porque es respaldada por los especialistas, así como la necesidad de formación del profesorado para trabajar en equipo.

Palabras clave: Consumo de drogas; estudiantes adolescentes; propuesta educativa; resiliencia.

\footnotetext{
"Correspondencia: alexandra.cango@educacion.gob.ec (A. Cango).

a (iD) https://orcid.org/0000-0002-1095-8963 (alexandra.cango@educacion.gob.ec).

b iD https://orcid.org/0000-0002-9103-9714 (nsuarez@unibe.edu.ec).
} 


\title{
Drug consumption in Ecuadorian students. An alternative for pre- vention and resilient development of students from the school
}

\author{
ABSTRACT
}

Drug use among Ecuadorian adolescent students is a growing phenomenon that manifests itself at the cognitive, social and health levels. The background shows it as a multifactorial phenomenon, linked to other addictions, with greater presence in areas of extreme poverty and disadvantaged social environments. Being the school one of the centers that can contribute substantially in the decision making of adolescents who live in areas of drug cultivation, trafficking and consumption, there is still insufficient educational work to win the advance of this scourge. The problem was identified through a mixed cross-sectional study. A total of 360 surveys were applied to students between the ages of 12 and 15 years old and an interview was conducted with the academic authority of the educational institution where the research was carried out and the officer of the Student Counseling Department. More than 50\% have at some point consumed drugs and there is evidence that leads to drug dependence in adolescents and, consequently, to the deterioration of their health. Given these findings, the objective is to diagnose the contextual situation of the educational unit under study and offer a system of extracurricular activities that seeks to prevent drug use and the resilient development of students. The feasibility of the proposal is concluded, because it is supported by specialists, as well as the need for teacher training to work as a team.

Keywords: Drug consumption; adolescent students; educational proposal; resilien.

\section{Introducción}

En Ecuador, el consumo de drogas es uno de los más altos de América Latina, con una incidencia de 51\%; superior a la de Chile, Uruguay y Perú (Loor, Hidalgo, Macías, García y Scrich, 2018). El fenómeno se revierte fundamentalmente en los jóvenes, uno de los grupos que más acuden a esas sustancias tóxicas de acuerdo con el Informe de la CONSEP (2005), que indicó que el 60,7 \% de la población realizó su primer consumo de alcohol entre los 15 y 19 años. En edades de la adolescencia y la juventud, las consecuencias de la drogodependencia son nefastas para la salud y la calidad de vida de los seres humanos, pues el organismo humano se encuentra en un proceso de maduración. Este resulta interrumpido por sustancias externas y tóxicas que no siempre se pueden rechazar, sobre todo si la tendencia es a incrementar la ingestión de ellas (Montero et al., 2020).

Los adolescentes ecuatorianos suelen consumir con mayor frecuencia drogas como la marihuana, un alucinógeno menor que actúa como depresor y desorganizador del sistema nerviosos central; el hachís, porro o canuto, contiene dosis de THC superior al de la marihuana sin procesar (Riofrío y Nascimento, 2010; ONU, 2010); el alcohol, sustancia que produce efectos inmediatos como euforia, desinhibición y sensación repentina de bienestar (Meneses, 2020; Rial, Golpe, Barreiro, Gómez y Isorna, 2020); tranquilizantes utilizados para el tratamiento del estrés, la ansiedad o conciliar el sueño; el éxtasis, que tiene efectos antidepresivos y sirve para aumentar la resistencia física; la heroína, empleada para incrementar la resistencia física del cuerpo humano, y el LSD (dietilamida del ácido lisérgico), una droga altamente 
alucinógena y que contiene sustancias químicas que alteran el estado de ánimo (Cortaza, Villar y Puig, 2012).

El uso y abuso de las drogas se encuentra asociado con la reducción de los niveles de desempeño físico-afectivo en edades tempranas, debido a las afectaciones directas que genera en el cerebro: un menor volumen en el hipocampo y en la corteza pre-frontal. Resulta perceptible el pobre desarrollo a nivel verbal, atencional y viso-espacial (Costa, Araújo, Silva y Luck, 2013; Júnior y Gaya, 2015; Meneses, 2020). En el orden social, además de los problemas de salud, el comportamiento de las personas genera conflictos escolares, legales, familiares y afectivos (Júnior y Gaya, 2015); porque los sujetos adictos suelen involucrarse con situaciones violentas, prácticas sexuales de riesgo, accidentes de tráfico (Rial et al., 2020).

Una de las cuestiones que influyen en la drogodependencia es la permisibilidad legal y despenalización del consumo de drogas, que en el caso ecuatoriano permite que un consumidor, sin incurrir en ningún delito, puede portar cierta cantidad en gramos de sustancias como: $10 \mathrm{~g}$ de marihuana, $2 \mathrm{~g}$ de pasta base de cocaína, $1 \mathrm{~g}$ de clorhidrato de cocaína, $0,1 \mathrm{~g}$ de heroína, 0,015 g de meta, 3.4 g de metilendioxifenetilamina, $0.015 \mathrm{~g}$ de éxtasis y $0.040 \mathrm{~g}$ de anfetaminas (CONSEP, 2005).

De ahí la necesidad de prevenir no solo desde una política para evitar el abuso del consumo de las drogas a nivel de país, sino en ayudar al estudiante a saber elegir y a decidir en un mundo donde la presencia de las drogas es algo natural con la puesta en práctica de estrategias efectivas (Montero et al., 2020; Sánchez y García, 2008). Precisamente, las instituciones educativas son lugares propicios en donde se puede influir en el comportamiento de los adolescentes a través de la prevención (Rivadeneira et al., 2020; Villarreal, 2020). De ahí se derivan los objetivos de diagnosticar la situación contextual de la unidad educativa en estudio y ofrecer un sistema de actividades extracurriculares que busca la prevención del consumo de drogas y el desarrollo de actitudes de resiliencia del alumnado.

\section{Antecedentes}

Las drogas, de acuerdo con la Organización Mundial de la Salud (OMS, 1994), constituyen sustancias terapéuticas o no que, introducidas en el organismo humano producen alguna alteración del funcionamiento natural del sistema nervioso central del individuo; y además son susceptibles de crear dependencia, ya sea psicológica, física o ambas. La Organización Mundial de las Naciones Unidas (ONU), indica que, desde la crisis económica de 2008, el consumo mundial de drogas ha aumentado en un 30\%; una década después se reportaba alrededor de 269 millones de consumidores que incluyen tanto a mujeres como a los hombres (Plazas, 2020).

El consumo de drogas es un resultado no solo de la voluntad de los sujetos que acuden a ella, sino que se encuentra acentuado por estructuras sociales que incentivan la dependencia hacia las sustancias tóxicas y psicoactivas. En primer lugar, la carencia del apoyo debido a la disfuncionalidad familiar; la influencia de amigos y circunstancias de un entorno violento, pobre y marginal. En segundo lugar, la falta de cultura respecto a las desfavorables consecuencias de las drogas (Loor et al., 2018).

Pavón (2020) demostró que estas situaciones no solamente se generan en las ciudades, sino que se revelan como conflictos vigentes en zonas rurales, y aunque se produce con mayor frecuencia en los hombres, también las mujeres resultan vulnerables al problema (Moraes et al., 2020; Romo, 2020). Ello trae consigo el incremento de la prostitución y explotación sexual de ambos sexos (Meneses, 2020); en otros casos ocurren las anomalías de los niños por el consumo de la madre de sustancias tóxicas (Moraes et al., 2020) y conductas antisociales en edades de la adolescencia (Costa et al., 2013; Montero et al., 2020). 
Villarreal (2020) indicó en su estudio que hay varios factores en los que la educación puede contribuir para paliar el fenómeno del consumo de drogas, en primer lugar, favorecer una conciencia acerca de la necesidad de construir políticas saludables, es decir, crear ambientes que beneficien la calidad de vida; reforzar la acción comunitaria; reorientar los servicios de salud hacia la prevención de las problemáticas sanitarias; y muy importante, el desarrollo de habilidades personales, más que de etiquetar a los estudiantes.

Hoy la resiliencia constituye una de las actitudes que más se trabaja en el área de la salud para prevenir conductas como la drogodependencia. De acuerdo con el estudio de García (2015), el concepto surge del estudio de la etiología y la psicopatología, fundamentalmente. Cortez (2010) la identifica como la capacidad de una persona para recobrarse de la adversidad fortalecida y dueña de mayores recursos. Se trata de un proceso activo de resistencia, autocorrección y crecimiento como respuesta a las crisis y desafíos de la vida. La resiliencia se encuentra vinculado con el patrón relacional, que incluye el valor de la cercana relación de confianza y de amplia red social; el patrón situacional, que incluye habilidades de valoración cognitiva, habilidades de solución de problemas, y atributos que indican una capacidad para la acción frente a una situación; el patrón filosófico, que es la creencia de que la vida tiene un propósito, que cada persona tiene un camino en la vida que es único y que es importante mantener una perspectiva equilibrada de la propia vida (Amar, Kotliarenko y Abello, 2003; Becoña, 2006).

Al respecto, Trigozo, Alvan y Santillan (2016) propone el desarrollo del modelo de las 5 "C" desde la escuela, que consiste en generar acciones que activen la Competencia: referido a la capacidad del adolescente para mostrar un buen desempeño en diversos ámbitos de la vida; la Confianza, que representa un sentido interno de valoración global positiva de uno mismo y de autoeficacia: autoestima, identidad y autoeficacia; la Conexión, referido a los vínculos positivos con personas (familia, iguales, adultos) e instituciones que se reflejan en intercambios bidireccionales; el Carácter: que trata del respeto por las normas sociales y culturales, que se relaciona con el autocontrol y la ausencia de problemas externos o conductuales; el Cuidado y Compasión: que abarca un sentido de simpatía y empatía e identificación con los demás, todos aspectos de gran importancia en la labor educativa y de pares entre adolescentes.

Es muy frecuente encontrar en el profesorado miedos, inseguridad para enfrentar esta problemática, pero sobre todo la ausencia de programas formativos que contribuyan a la promoción de salud en el ámbito escolar (Rivero, Suárez y Fernández, 2017). Esta alternativa de intervención que se presenta desde la Educación da pautas a los docentes que se enfrentan a esta problemática. Para ello, luego de haber presentado un breve panorama conceptual y del estado de la cuestión, resulta pertinente en un principio diagnosticar el nivel de consumo de estupefacientes en un centro educativo de la región costa ecuatoriana que presenta una problemática común en la zona en estudio para, a partir de ahí, proponer un sistema de actividades extracurriculares dirigido a la prevención del consumo de drogas y el desarrollo de actitudes de resiliencia del alumnado.

\section{Metodología}

La investigación identifica dos momentos, el primero una captura transversal de la problemática y el segundo ofrecer luego una solución contextual. En este caso, implica la contribución de prácticas de prevención de la drogodependencia en adolescentes. Esta perspectiva conllevó un enfoque mixto secuencial de investigación (cuantitativo-encuestas y el cualitativo-entrevistas) y el estudio alcanza un nivel exploratorio, lo cual sienta las bases para establecer una propuesta de intervención educativa en la fase de planeación. De esta forma generar una gestión escolar que incremente la efectividad de la labor educativa. 


\subsection{Participantes}

El estudio resultó viable realizarlo en una Unidad Educativa de la provincia de Santo Domingo de los Tsáchilas, en Ecuador por la posibilidad de acceso a los datos. Al ser la autora principal de este trabajo, docente de la institución y enfrentar la problemática diariamente, se crearon niveles de empatía importantes con los estudiantes para recoger este tipo de datos. Interesa centrarse en la detección del cómo se manifiesta el problema del consumo de drogas de los adolescentes. El muestreo se realizó en la población de adolescentes con edades comprendidas entre los 12 y 15 años de ese centro escolar.

Por tanto, la muestra resultó ser no probabilística e intencional, participando la totalidad de 400 estudiantes pertenecientes a la matrícula de los nueve cursos de Educación Básica Superior de los paralelos de octavo, noveno y décimo año. Resulta importante destacar que, del total, el número de cuestionarios descartados fue de 40 y se excluyeron porque no respondieron a todos los ítems propuestos, por tanto, el número total de participantes fue de 360 . Además, se realizaron dos entrevistas a la máxima autoridad de la escuela y a una especialista del Departamento de Consejería Estudiantil (DECE).

\subsection{Instrumentos}

Para la investigación se diseñaron dos instrumentos, el primero fue una encuesta que contó con 12 ítems, cuyo Alfa de Cronbach calculado fue de 0,8; lo cual es una consistencia interna buena. Con ella se recolectaron datos demográficos, y además se supo sobre el conocimiento de drogas y entorno escolar (P1, P2, P3); las afectaciones a la salud y a las relaciones sociales que trae consigo su consumo (P4; P10); particularidades del consumo (P5, P6, P7), entorno familiar y comunitario (P8, P9, P11) y actividades de prevención preferidas por los estudiantes (P12), todo ello desde los fundamentos teóricos que facilitan luego procesos de prevención y la potenciación de la resiliencia. La Tabla 1 muestra el cuestionario aplicado. En el preámbulo de este se informa al estudiante sobre el objetivo que pretende alcanzar el estudio que se quiere realizar, que han sido seleccionados al azar para participar y que la información que suministre será confidencial. Asimismo, se presentan las indicaciones para su correcto completado.

\section{Tabla 1}

Cuestionario aplicado.

\begin{tabular}{|c|c|}
\hline Ítems & Opciones de respuesta \\
\hline Datos demográficos & Año__ Jornada \\
\hline $\begin{array}{l}\text { 1. Marque las sustancias psicotrópicas o drogas } \\
\text { que conoces. }\end{array}$ & $\begin{array}{l}\text { cocaína_marihuana__ heroína__ éxtasis_ } \\
\text { cripy_otra_ninguna_ }\end{array}$ \\
\hline $\begin{array}{l}\text { 2. Marque a las personas que conoces que hayan } \\
\text { probado/consumido algún tipo de droga. }\end{array}$ & $\begin{array}{l}\text { amigos de la escuela__vecinos de lugar donde } \\
\text { vives__ familiar_ otros }\end{array}$ \\
\hline $\begin{array}{l}\text { 3. Marque el lugar/ocasión que has visto consu- } \\
\text { mir drogas. }\end{array}$ & $\begin{array}{l}\text { eventos sociales__ conciertos__ horario de re- } \\
\text { ceso dentro de la institución_fuera de la institu- } \\
\text { ción__en el hogar_ en ningún lugar_ }\end{array}$ \\
\hline $\begin{array}{l}\text { 4. Marque los órganos que se afectan con el con- } \\
\text { sumo de drogas o alcohol. }\end{array}$ & $\begin{array}{l}\text { cerebro_estómago__hígado__pulmones__gar- } \\
\text { ganta_ninguno_. }\end{array}$ \\
\hline $\begin{array}{l}\text { 5. Marque si has probado alguna de estas drogas/ } \\
\text { alcohol al menos una vez. }\end{array}$ & $\begin{array}{l}\text { cocaína_marihuana__ } \\
\text { cripy_alcohol_otra__ninguna__ nina }\end{array}$ \\
\hline $\begin{array}{l}\text { 6. Marque con qué frecuencia pruebas o consu- } \\
\text { mes alguna de estas drogas/alcohol. }\end{array}$ & $\begin{array}{l}\text { nunca_ al menos una vez _ siempre _a veces } \\
\text { _ocasionalmente__ }\end{array}$ \\
\hline
\end{tabular}




\begin{tabular}{|c|c|}
\hline $\begin{array}{l}\text { 7. Marque la razón por la que consumes drogas/ } \\
\text { alcohol. }\end{array}$ & $\begin{array}{l}\text { Problemas familiares_curiosidad_celebridades } \\
\text { que consumen y admiras_socializar con ami- } \\
\text { gos_medios de comunicación__otras__ }\end{array}$ \\
\hline $\begin{array}{l}\text { 8. Marque de las siguientes personas quién vive } \\
\text { contigo en hogar. }\end{array}$ & $\begin{array}{l}\text { vivo solo__ padre__ padrastro__ madre _ ma- } \\
\text { drastra__ hermanos_abuelos_ otros que no } \\
\text { somos familia_ }\end{array}$ \\
\hline $\begin{array}{l}\text { 9. Marque con qué frecuencia en el hogar se } \\
\text { preocupan por si fumas, consumes alcohol u } \\
\text { otras drogas. }\end{array}$ & $\begin{array}{l}\text { siempre_casi siempre_a veces__casi nunca__ } \\
\text { nunca__ }\end{array}$ \\
\hline $\begin{array}{l}\text { 10. Marque si como consecuencia del consumo } \\
\text { de drogas has sentido. }\end{array}$ & $\begin{array}{l}\text { Rechazo de los demás_enojo injustificado, riñas } \\
\text { _poder sobre los demás__aceptación de los de- } \\
\text { más_estado de bienestar. }\end{array}$ \\
\hline 11. Marque si venden cerca de tu casa. & cigarrillos__alcohol__drogas__ no venden \\
\hline $\begin{array}{l}\text { 12. Marque el tipo de actividad en que participa- } \\
\text { rías para aprender más sobre el consumo de dro- } \\
\text { gas alcohol, sus efectos y cómo prevenirlas. }\end{array}$ & $\begin{array}{l}\text { películas_ teatro__ juegos _ intercambios con } \\
\text { otros estudiantes__ concursos__ }\end{array}$ \\
\hline
\end{tabular}

Fuente: Elaboración propia.

El segundo fue una entrevista semiestructurada que contó con siete ítems, dirigida a la principal autoridad académica (vicerrectora) de la escuela, pues se entiende que debe tener un conocimiento amplio sobre las problemáticas que ocurren dentro de su centro escolar y a la especialista del Departamento de Consejería Estudiantil (DECE), centro independiente de la escuela, pero dirigido por el Ministerio de Educación para el diagnóstico y orientación a las escuelas. En ambos casos se indagó acerca de: la percepción respecto a las problemáticas generadas por el consumo de drogas como un problema dentro de la institución, los datos referidos al consumo de sustancias psicoactivas en el centro, qué sustancias o drogas son las más conocidas y usadas, identificación de estudiantes o cursos con mayor tendencia a consumir drogas, causas más comunes y consecuencias más visibles entre los que consumen, formas o acciones efectuadas para prevenir esta problemática social, finalmente, qué recomendarían para evitar la exposición y consumo de estupefacientes por parte de los estudiantes del plantel.

\subsection{Procedimiento}

La recogida de los datos se efectuó en las propias instalaciones del centro educativo objeto del estudio, para ello se contó con la autorización de la rectora y la participación voluntaria de los estudiantes bajo consentimiento informado y dos docentes que apoyaron en la aplicación de encuesta. La encuesta se completó durante el primer quimestre del año 2019 de manera individual y física en papel, El análisis de los datos se realizó con el apoyo del programa Excel, facilitando la interpretación estadística de la información recabada.

En el caso de las entrevistas, también se localizaron dos espacios de la Unidad Educativa con tranquilidad para poder garantizar la concentración de los entrevistados. El diálogo fluyó de manera amena y respetuosa, con la menor intervención posible de las opiniones subjetivas del entrevistador para contribuir a una mayor objetividad de los datos resultantes. El diálogo se grabó con el conocimiento y autorización de los dos profesionales entrevistados, para posteriormente transcribirse y proceder a su interpretación. 


\section{Resultados}

\subsection{Resultados de la encuesta}

\subsubsection{Conocimiento de las drogas y entorno escolar}

De los 360 estudiantes participantes de la encuesta, el 58\% son representantes del género femenino. Este grupo de alumnos tiene edades que oscilan entre los 12 y 15 años, y cursan los años de octavo hasta el décimo; lo cual resulta un aspecto importante en este estudio, teniendo en cuenta la necesidad de prevenir el consumo de drogas justamente en el periodo de vida donde existen más probabilidades de iniciar el uso de las sustancias psicoactivas y tóxicas y donde también provoca daños irreversibles por la propia etapa en la que aún no ha concluido su desarrollo físico y emocional. Así, con respecto a la pregunta relacionada con el conocimiento del tipo de droga, los resultados encontrados pueden verse en la Figura 1.

\section{Figura 1}

Tipos de drogas que se conocen.

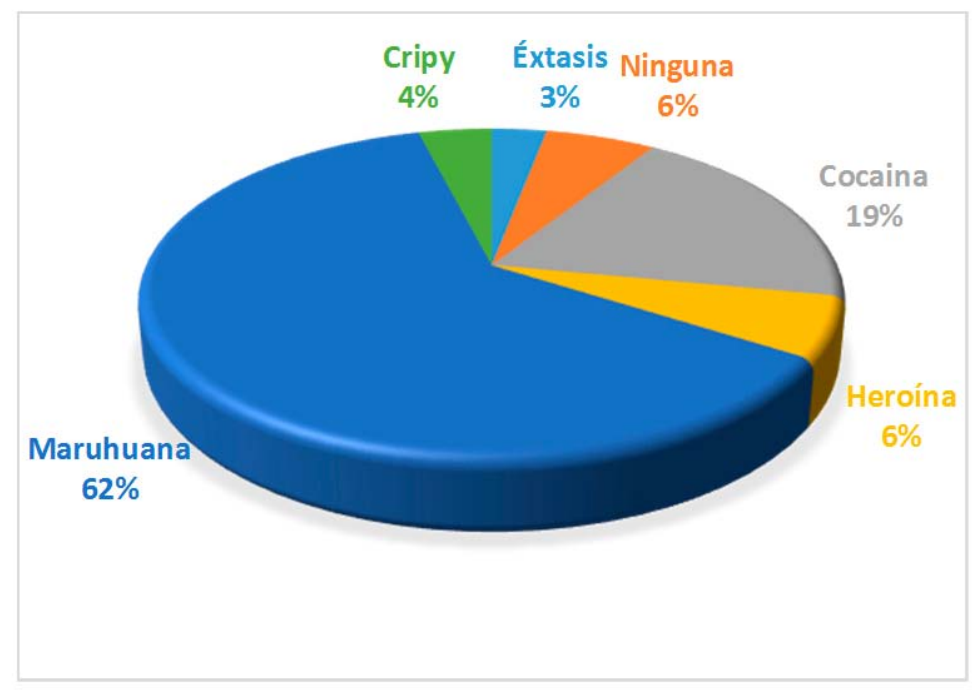

Fuente: Encuesta aplicada a estudiantes.

De acuerdo con las respuestas de los estudiantes, el 94\% mencionaron que conocen las drogas mencionadas, siendo la marihuana la más conocida, tanto en hombres como mujeres. El 6\% que dijo no conocer ninguna son mujeres. Este dato es indicador no solo de que ya a esas edades los jóvenes ecuatorianos saben reconocer a las sustancias psicoactivas, sino también de que resulta evidente que la droga se encuentra presente en el ámbito de desarrollo de los adolescentes.

Buscando comprender este dato se indagó sobre el contacto que tienen los estudiantes con personas que prueban/ consumen drogas. No fue extraño entonces que el $91 \%$ conocieran a alguna persona con esta condición. El grupo más conocido es el de amigos de la escuela $50 \%$, luego el del lugar donde viven, $25 \%$, del grupo familiar, $14 \%$ y un $2 \%$ refirió a otros. Este resultado puede estar asociado a que la Unidad Educativa está ubicada en uno de los sectores que acoge a estudiantes de sectores con alta vulnerabilidad socioeconómica, es decir, altos índices de pobreza, marginación, violencia, donde el empleo de sustancias tóxicas puede resultar conveniente para evadir los problemas sociales que enfrentan. 
Este aspecto se corrobora precisamente con la interrogante que indaga sobre el lugar donde han visto consumir la droga; más de la mitad, $53 \%$, han presenciado el consumo fuera de la institución educativa; mientras que un $25 \%$ lo vio en conciertos, un $13 \%$ en los recesos de horas clases, el $8 \%$ en eventos sociales y un $1 \%$ en otros estudiantes. De modo que se puede concluir que el consumo de drogas se manifiesta dentro y fuera de la institución educativa.

\subsubsection{Afectaciones a la salud y a las relaciones sociales que trae consigo su consumo}

En el ítem que interroga por las afectaciones a la salud que trae consigo el consumo de drogas o alcohol, la mayoría de los alumnos opina que el mal hábito afecta su cerebro, 41\%; al estómago, el 19\%, a los pulmones, el 17\%, la afectación al hígado, el 13\% y a la garganta, el $10 \%$. Un dato relevante de la encuesta permite conocer que a pesar de que un significativo grupo de estudiantes ( $74 \%$ de los encuestados) conoce que el consumo de algún tipo de droga o alcohol ocasiona problemas a la salud, no todos son capaces de evitar el hábito o la ingestión ocasional de esas sustancias. En el ítem que trata de que los estudiantes reconozcan los efectos de las drogas en sus relaciones, el $80 \%$ indicó la sensación de bienestar que le provoca y en menor medida a riñas y al poder sobre los demás. Esto puede estar atribuido a la baja percepción de riesgo de iniciarse en este hábito o probablemente porque las drogas y alcohol provocan dependencia y, al inicio, una sensación de bienestar por evasión de la realidad.

\subsubsection{Particularidades del consumo}

Al tratar de conseguir un dato más concreto sobre el consumo, se revelan coincidencia con la pregunta relacionada con el conocimiento de estas. Ello se confirmó en la pregunta que busca conocer la frecuencia del consumo. Los datos extrémales indicaron que, el $55 \%$ de los encuestados han probado o experimentaron al menos una vez las drogas/alcohol y únicamente, el $4 \%$ nunca ha consumido ninguna de estas sustancias, solo existe un margen de un 2 $\%$ que, conociéndolas, no las consume. La figura 2 muestra el detalle de la frecuencia.

\section{Figura 2}

Frecuencia de la experimentación de consumo drogas.

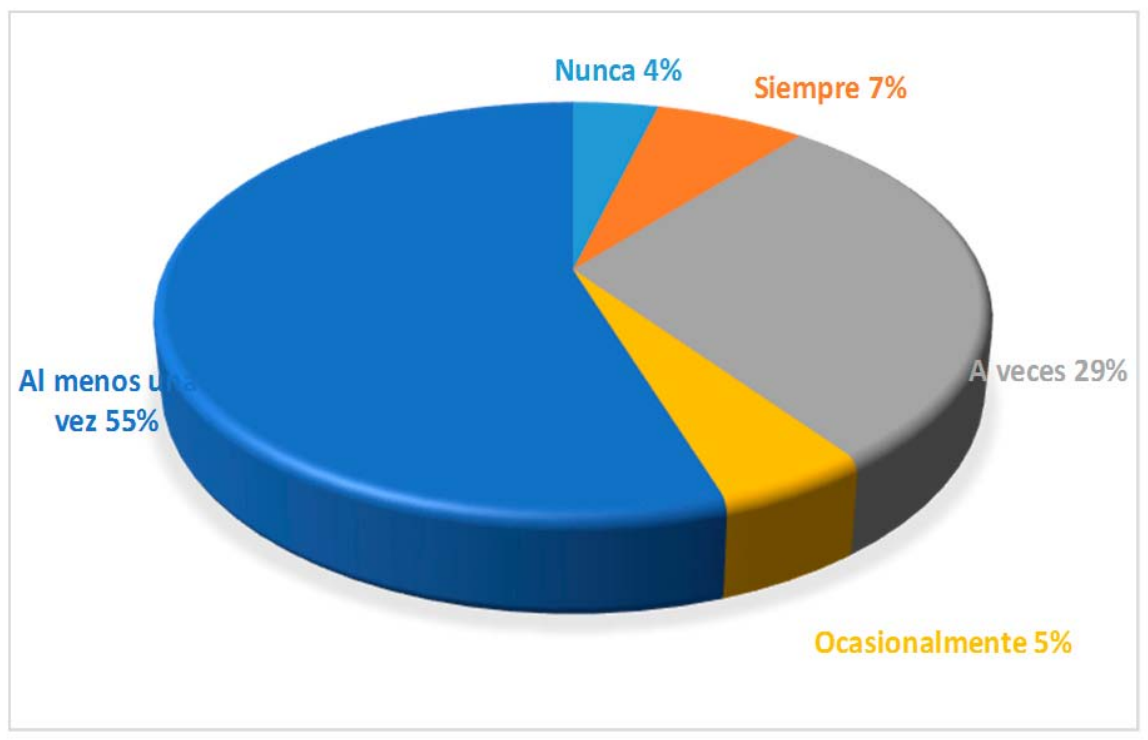

Fuente: Encuesta aplicada a estudiantes. 
Seguidamente, se muestran las razones del consumo. Los problemas familiares acogen a más de $50 \%$ de los estudiantes, luego inciden la red de amistades; la tendencia a admirar celebridades que consumen, la influencia de los medios de comunicación y la curiosidad. La figura 3 muestra las diferencias en la influencia de cada uno de estos factores.

\section{Figura 3}

Factores causales que inciden en el consumo de droga en adolescentes.

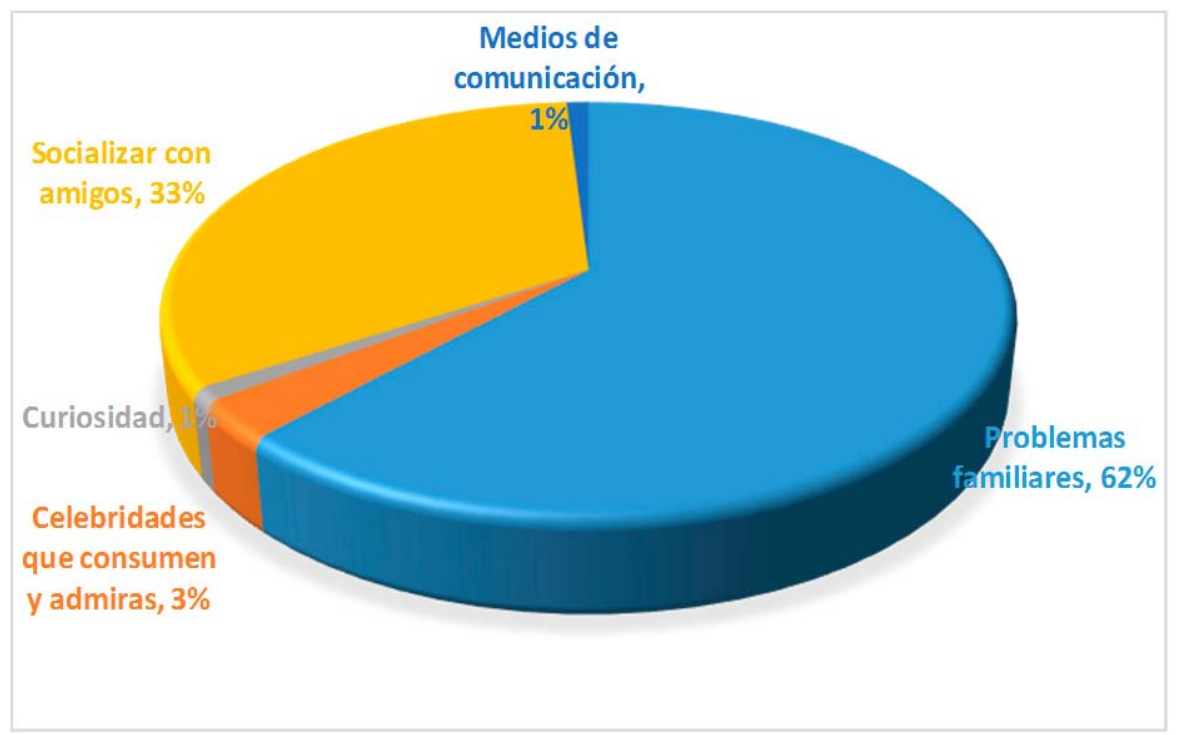

Fuente: Encuesta aplicada a estudiantes.

Este resulta un saldo muy desfavorable, en tanto, la ingestión de drogas en edades de la adolescencia está relacionada con las prácticas adictivas de los jóvenes y adultos, debido a la dependencia que se genera en el organismo, sobre todo en el cerebro, del consumo de una sustancia que fuerza la actividad física, las manifestaciones alucinógenas, así como la sensación de bienestar. Al mismo tiempo se degradan las competencias cognitivas y afectivas de los sujetos y las capacidades del cuerpo humano. Aquello que proviene del entorno social comienza a verse como un fenómeno natural por parte de los adolescentes que se tornan incapaces de percibir el riesgo de esta práctica.

\subsubsection{Entorno familiar y comunitario}

Creemos que los resultados de los ítems anteriores están muy asociados a la atención que presta la familia a problemática, pues en el $39 \%$ de los hogares casi nunca se preocupan si consumen o no drogas, no se menciona este tipo de problemáticas sociales, siendo fundamental en los jóvenes al tener edades en donde necesitan conocer sus entornos. Los cambios que se manifiestan al transitar de niños a adolescentes son difíciles de controlar por los padres, por lo que es fundamental el acompañamiento de su familia y la aclaración de sus inquietudes, de lo contrario, se advierte el riesgo de conocer este tipo de prácticas fuera de la familia como lo muestra este estudio, con personas equivocadas que inciden desfavorablemente en las conductas de las personas jóvenes. Todo ello debido a que el $62 \%$ de los casos se trata de estudiantes que viven con solo uno de los padres o familiares.

A su vez, otra causa de este comportamiento del fenómeno radica precisamente en las características del entorno comunitario y social en el cual se involucran los jóvenes, pues el 34\% 
mencionaron que cerca de sus casas se expenden drogas. En la actualidad, resulta inevitable la venta de drogas legales como el alcohol y tabaco en las tiendas, sin comprobar si el consumidor es mayor de edad. En el contexto en estudio, la situación resulta más compleja porque la escuela se nutre de adolescentes que viven en sectores con alta precariedad socioeconómica, sectores considerados de riesgo, por tal razón es más probable que estén inmersos primero en el expendio como sustento económico y luego en consumo de algún tipo de droga.

\subsubsection{Actividades de prevención preferidas por los estudiantes}

A pesar de que se comprobó que en la escuela y sus alrededores se consume drogas, un saldo positivo de estas respuestas radica en el hecho de que el centro educativo no se contempla como un espacio que influye directamente en la práctica de consumo de sustancias tóxicas, aun cuando el $33 \%$ de causas tiene que ver con socializar con amigos del colegio, pero lo hacen en actividades extraescolares; lo cual resulta favorable para el presente estudio que pretende desde la orientación educativa fomentar una conducta resiliente en el alumnado con el que se pueda interrumpir definitivamente o evitar la ingestión de sustancias adictivas.

Otro resultado alentador que muestran los datos de la encuesta es que la mayoría de los estudiantes, el 96\%, indicó que siempre participarían en actividades para la prevención del consumo de drogas. La figura 4 muestra mayor énfasis en aquellas relacionadas con el cine y el intercambio entre iguales a través de las tecnologías. Estos resultados significan una verdadera oportunidad para convertir a la escuela en el principal escenario de acciones educativas efectivas para potenciar la resiliencia y con ello reducir o eliminar definitivamente el consumo de drogas.

\section{Figura 4}

Preferencias de los estudiantes de actividades preventivas de la drogodependencia.

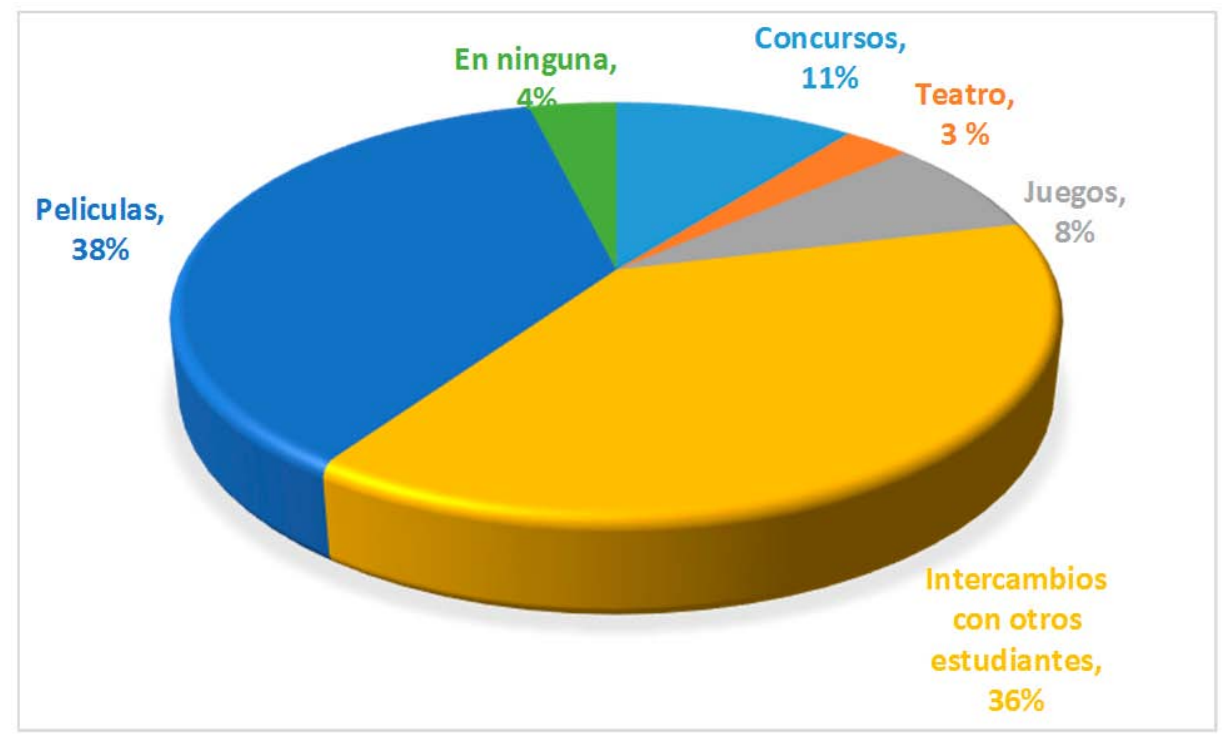

Fuente: Encuesta aplicada a estudiantes. 


\subsection{Resultados de las entrevistas}

\subsubsection{Institución educativa: barrera preventiva del consumo de drogas en adolescentes}

Con la finalidad de conocer si la autoridad académica era consciente de la existencia del hábito de consumo de droga en los estudiantes del plantel, la dinámica utilizada fue la de realizar preguntas semiestructuradas a la vicerrectora que generen respuestas desde su perspectiva, logrando un proceso de interacción que además permitió hacer preguntas complementarias a las previstas según se avanzó en la entrevista. En lo adelante se explicitan las opiniones más relevantes.

Uno de los resultados más significativos fue la respuesta acerca de su opinión sobre el problema de las drogas en la institución. La directiva expresó que: "de manera general el consumo de drogas en los estudiantes va en aumento cada año, lo que causa problemas de comportamiento, déficit de atención, bajos rendimientos, inasistencias a las horas de clase, entre otros" (comunicación personal, 2020). Señaló, además "si bien abiertamente no se han reportado casos específicos de consumo de drogas en la institución, se sospecha que el problema existe debido a la actuación de los jóvenes: el incremento de deserciones, conflictos entre pares, bajo rendimiento académico y manifestaciones físicas que así lo indican".

Según la vicerrectora, entre las acciones a tomar en cuenta para prevenir esta problemática social se encuentra el trabajo con la familia y el acompañamiento de la comunidad educativa en el enfrentamiento a ese flagelo. Alude que "se tiene un trabajo conjunto con el Departamentos de Consejería Educativa (DECE) y ellos son los encargados de realizar las campañas orientadas por el Ministerio de Educación con respecto a la prevención, pero esto no es suficiente" (comunicación personal, 2020).

La especialista del DECE, por su parte, señaló que tampoco este centro cuenta con algún reporte de estudiantes que ingieran o consuman algún tipo de estupefaciente. No obstante, cabe recalcar que su presencia es la institución es de poco menos de dos meses y recién estaba conociendo el entorno educativo. Advierte que, en sus primeras pesquisas, la sospecha de estudiantes que estén inmiscuidos en algún tipo de consumo la reconoció por el enrojecimiento de los ojos, pupilas dilatadas, frecuencia con la que se refrían y conductas agresivas en extremo.

De acuerdo con la experiencia de la especialista del DECE existen varias causas del incremento de la incidencia del uso de drogas y alcohol, entre ellas, el abandono familiar de los adolescentes, la relación no cercana entre padres e hijos en muy común por las altas tasas de separación de los padres, escaso afecto paternal, desconocimiento sobre cómo afrontar frustraciones, resolver problemas y controlar impulsos. Por otro lado, se vinculan los fracasos escolares, el abuso sexual, la violencia, no tener un hogar funcional. Menciona también que las drogas más consumidas son el alcohol, tabaco y marihuana, lo cual se relaciona con las dosis permitidas y esto en su criterio es la puerta de entrada.

Según la especialista, la estrategia para contrarrestar esta problemática social debería consistir en la prevención integral, un "proceso que brinda espacios para el desarrollo de las capacidades del sujeto, familia y comunidad, enfocado a disminuir los factores de riesgo y fortalecer los factores protectores, garantizando el respeto a los derechos humanos y con la participación de todos los actores" (comunicación personal, 2020).

El diagnóstico de la situación permite finalmente presentar la propuesta de intervención educativa, basada en un trabajo intersectorial y multidisciplinario en donde se generan espacios de participación para brindar la oportunidad a los estudiantes de fortalecer habilidades y destrezas, que permitan el desarrollo de los aspectos positivos del ser humano, estableciendo un ambiente armonioso para el desarrollo del mismo. 


\section{Descripción de la propuesta}

Sistema de actividades extracurriculares potenciadoras de la resiliencia y la prevención del consumo de drogas en estudiantes de Educación Básica Superior.

\subsection{Definición y características generales}

Un sistema de actividades extracurriculares es una estrategia educativa que propicia el tratamiento, con carácter preventivo, del problema del consumo de drogas en la etapa de la adolescencia, en tanto, favorece sentimientos de motivación y el aprendizaje, a través de acciones que si bien van dirigidas a incrementar los conocimientos sobre las graves consecuencias de la drogodependencia, la forma dinámica y atractiva de efectuarse garantiza la atención y estimula la participación juvenil en ese tipo de actividades.

Entiéndase por sistema al conjunto ordenado de elementos o componentes interrelacionados, interdependientes, interactuantes con la finalidad de lograr los objetivos de un plan (Cajizo, 1975; Chiavenato, 2017). El sistema de actividades extracurriculares se define como un conjunto de tareas que se complementan entre sí, con un solo propósito de contrarrestar aquella deficiencia identificada y buscar los mecanismos de llegar a un solo fin, con el fortalecimiento y la cooperación de todos. Las acciones extracurriculares suelen fomentar un espacio diferente a la formalidad que transcurre dentro del aula y la confianza de los estudiantes en ellos mismos (UNESCO, 2018). Están dirigidas además a la estimulación de la satisfacción de los alumnos por mediación del aprendizaje colaborativo y significativo que favorecen un mejor comportamiento académico, social y comunitario. Cada una de las actividades extracurriculares están concatenadas entre sí de forma sistémica en las cuales unas dependen de las otras y tiene un orden para vincular empáticamente a los estudiantes con la resiliencia para dejar el consumo de drogas, tal como se presenta en la Tabla 2.

\section{Tabla 2}

Actividades versus impacto.

\begin{tabular}{|l|l|}
\hline \multicolumn{1}{|c|}{ Actividades Extracurriculares } & \multicolumn{1}{c|}{ Aspectos Resilientes que potencian } \\
\hline Cine Debate & Autoestima \\
Teatro & Aceptación \\
Focos Group & Autonomía \\
Foro de Discusión online & Autosuficiencia e independencia \\
Concurso & Autoconocimiento \\
\hline
\end{tabular}

Fuente: Elaboración propia.

Los beneficiarios directos de la propuesta educativa son los estudiantes de Educación Básica Superior, al ser adolescentes con edades comprendidas entre los 12 y 15 años, los cuales experimentan cambios biopsicosociales. La comunidad educativa debe estar inmersa en la propuesta al ser quienes lideren este cambio. Su impacto social es reducir de alguna manera los porcentajes de alumnos que consumen drogas a través de estrategias de resiliencia fundamentales para una vida sana en consonancia con la Matriz Estratégica Institucional del 2017 hasta el 2021- MINEDUC.

La importancia de la propuesta reside en la posibilidad de convertir al centro educativo en un espacio de protección integral e inclusiva de estudiantes y al mismo tiempo de formación de estudiantes promotores pares que luego incidan sobre los demás estudiantes, ello le impregna el carácter preventivo. Otra parte importante de esta propuesta señala la sensibilización de docentes y comunidad educativa sobre rutas y protocolos a seguir ante casos de adicciones; así como generar una cultura escolar respetuosa de las diferencias y consolidada en equipos de autogeneración de soluciones. 
La pertinencia de un proyecto radica en que es un primer paso en una institución donde todos conocen el problema, pero pasa como "desconocido". Para insistir en la necesidad de crear alianzas estratégicas con organismos del Estado o no gubernamentales para generar proyectos de fortalecimiento humano integral a una acción educativa concreta encaminada a reducir las prácticas adictivas. Por eso desarrollar una cultura resiliente en los estudiantes, de percepción del riesgo y de medidas preventivas, es esencial en esta propuesta desde las situaciones particulares del estudiantado que afectan directamente el normal proceso de aprendizaje y conlleva a problemas de salud y sociales.

\subsection{Estructura de la propuesta}

En tal sentido, el principal objetivo de la propuesta es:

\section{Objetivo general}

Diseñar un sistema de actividades extracurriculares para potenciar la resiliencia y prevenir el consumo de drogas en estudiantes de Educación Básica Superior.

\section{Objetivos especificos}

1. Organizar el sistema de actividades extracurriculares de forma sistémica para potenciar la resiliencia ante el consumo de drogas a nivel académico y social.

2. Estructurar de forma didáctica las actividades extracurriculares.

3. Evaluar por especialistas el sistema de actividades extracurriculares para potenciar la resiliencia.

\section{Marco legal}

La intervención se encuentra respaldada legalmente por el artículo 117 de la Ley Orgánica de Educación Intercultural, que prescribe: "La jornada semanal de trabajo será de cuarenta horas, de la siguiente manera: seis horas pedagógicas diarias cumplidas de lunes a viernes. El tiempo restante hasta cumplir las ocho horas diarias estará distribuido en actualización, capacitación pedagógica, coordinación con los padres, actividades de recuperación pedagógica, trabajo en la comunidad, planificación, revisión de tareas, coordinación de área y otras actividades contempladas en el respectivo Reglamento". Cada una de las acciones conjuntas al igual que las normativas busca el bienestar de los individuos en todo sentido.

Cabe mencionar que al trabajar con estudiantes de Básica Superior desde el Ministerio de Educación se sigue trabajando con los Proyectos Escolares y lo fundamental para la aplicación de la propuesta es utilizar esas horas durante un quimestre del año lectivo.

\section{Actividades}

\section{Actividad extracurricular 1: Cine debate}

Objetivo: Estimular el pensamiento reflexivo de los estudiantes sobre las consecuencias graves del consumo de drogas.

\section{Fase Inicial}

\section{Metodología:}

Se utilizará una metodología de aula invertida. La base orientadora antes de iniciar un debate sobre el tema consiste en observar un documental de 100 minutos titulado "La Verdad Sobre las Drogas: Gente Real, Historias Reales ${ }^{1}$.

1. Recuperado de https://www.scientology.es/how-we-help/truth-about-drugs/educational-film.html\#slide2. 
Para incrementar el compromiso y la implicación del alumno, de manera que construya su propio aprendizaje, se les solicita que observen atentamente y lo socialicen con sus compañeros desde una perspectiva comparada con su realidad antes de venir a clase.

Además, se les orienta sobre el espacio indicado para observar el documental; la coparticipación de los padres en la observación; debate en el espacio familiar y con los demás jóvenes que suscite preguntas acerca de las drogas.

Intencionalmente esta actividad, además sirve para conocer cómo piensan los jóvenes acerca de esta temática; identificación o no con alguno de los protagonistas del documental; estimular el debate habilitando momentos protagónicos de los estudiantes, con respecto a sus opiniones.

Fase desarrollo

Preguntas reflexivas

Durante el desarrollo el docente puede indagar sobre cómo transcurrió el proceso de observación orientado.

A través de un método de elaboración conjunta y de discusión poner a los estudiantes en situación de debate y reflexión.

¿Por qué el documental tendrá ese título y qué significado tiene?

¿Tiene relación con su vida o la de alguien cercano a usted?

¿Cuáles son los problemas centrales que se abordan?

¿Cuál es el final de los jóvenes protagonista que usan las drogas?

¿Los amigos influyen en la iniciativa de probar drogas?

¿Tenemos sueños que cumplir y que pasa si prefiero no cumplirlos por consumir drogas?

¿Nos podemos salvar de las drogas?

¿Tengo problemas en casa, cuáles?

¿Me han ofrecido droga, al igual que en el documental?

¿Tengo un Proyecto de Vida?

Dinámica: se utiliza la técnica Imagínate a ti mismo con la finalidad de trabajar con el componente de autoestima. El docente pide a los estudiantes pensar en el futuro y cómo se imaginan a sí mismos dentro de algunos años.

Fase Final

Como actividad de cierre se les recomienda ver películas en sus casas en compañía de sus padres relacionadas con el consumo de drogas en adolescente. El docente puede seleccionar cuidadosamente algunas como Trainspotting (1996), de Danny Boyle, The Basketball Diaries (1995), de Scott Kalver u otras que muestren historias reales de deterioro, pero también de superación. Algunas preguntas al terminar la observación de las películas pueden orientarse a:

¿Qué tipos de drogas consumen los protagonistas?

¿Qué justifica el consumo de drogas? ¿Cuál es el argumento que se utiliza para justificarlo?

¿Se muestran tratamientos de rehabilitación en la película? ¿Cuáles?

¿Se muestra alguna estrategia de reducción de daños?

Evaluación

Presentar fragmentos de la película a través de una obra de teatro recopilando la información ya observada. 
Temporalización: Se realiza dentro de las dos primeras semanas del quimestre.

Ubicación: En cada uno de sus hogares.

\section{Actividad extracurricular 2: Teatro}

Objetivo: Construir un espacio generador del desarrollo creativo en los estudiantes, que fomente la reflexión y la espontaneidad en revertir las vivencias observadas en películas relacionadas con el consumo de drogas en adolescentes con la aplicación en las formas de expresión.

Metodología: A partir de observaciones de películas de forma autónoma por los estudiantes pueden replicar el argumento o de una o crear su propio argumento a través de una obra de teatro que muestre fragmentos tensionantes y/o de reestructuración de conductas antidrogas. Se busca que a partir de la empatía (ponerse en los zapatos del otro) el estudiante logra con los diferentes personajes reflexiones y reestructure sus formas de actuación.

Nombre de la obra: Los estudiantes creativamente pueden elegir un nombre para su obra de teatro.

\section{Fase Inicial}

Los alumnos conjuntamente con los docentes reflexionan de forma global acerca de películas ya observadas. Una vez que han decidido qué película pueden escenificar o si es que harán una versión de la misma se organizan las actividades por cada uno de los grupos en lo que corresponde: Actuación, Dirección, Guion, Escenografía, Vestuario, Iluminación, Sonido, Montaje Técnico, selección de los personajes. El docente se autoprepara en algunas técnicas de presentación (Stanislavsky) para orientar al estudiante y finalmente crear las escenas.

Fase Media

Se desarrollan técnicas para trabajar en la implementación y argumento de la película.

Fase Final

Consiste en la evaluación de teatro. Se selecciona la alternativa de co-evaluación, mediante comentarios, preguntas y respuestas. Se pueden emplear varios instrumentos para valorar el desempeño: hoja de logros con la actuación por los grupos, lista de cotejo entre lo logrado y por lograr, hoja de autoevaluación, coevaluación y análisis. se realizan comentarios, reflexión y retroalimentación en relación a lo observado y se desarrollan fragmentos de ensayos de cada uno de los papeles que representan.

Temporalización: Se realiza dentro de la tercera y cuarta semana del quimestre.

Ubicación: Aula - salón múltiple.

\section{Actividad extracurricular 3: Focus group}

Objetivo: Identificar las diferentes estrategias de resiliencia a las drogas.

Metodología: Interacción Social de Vygostsky.

Fase Inicial

El docente a través del guion gráfico diseña los procesos de las actividades anteriores, se ayudará pidiendo a los jóvenes que escriban ideas en notas individuales de las experiencias ya realizadas, con ello hará una retroalimentación de todo. Conformará grupos de los distintos cursos a través de la dinámica "La Granja", utilizando tarjetas donde aparezca imágenes de diferentes animales; después de repartir, harán el ruido de cada animal hasta juntarse.

Los grupos focales se trabajará indistintamente por cada año de octavos, novenos y decimos de la enseñanza básica superior. Se definirá el propósito del grupo focal enfocado a la 
práctica de estrategias de resiliencia ante el consumo de drogas. Es importante identificar el rol del moderador.

\section{Fase Media}

Se definirá el cronograma para la realización de las secciones del focus group, detallando los recursos que se van utilizar para la actividad. Escribir la guía de discusión del grupo focal, permitirá organizarnos conjúntame con los grupos de trabajo, es necesario definir espacios para evitar posibles distractores y cumplir con la actividad. Posteriormente, se diseñará el plan de análisis, para asegurar la participación y fomentar la autonomía.

Fase Final

A partir del análisis de la información recolectada, se realizarán actividades de refuerzo para incidir sobre el Yo de cada participante de las categorías de factores resilientes: Yo tengo (apoyo externo); Yo soy (fuerza interior) y Yo estoy dispuesto a hacer (proyección), y Yo puedo (capacidades interpersonales y de resolución de conflictos):

Temporalización: Se realiza en el mes posterior.

Ubicación: Aula - salón múltiple.

\section{Actividad extracurricular 4: Foro de discusión online: ¿Cómo puedo ser promotor par?}

Objetivo: Interactuar entre el docente tutor y el alumno de manera asincrónica para generar la posibilidad de cómo hacer la labor de pares con amigos, vecinos en la reflexión sobre los efectos de las drogas en los jóvenes y las maneras de ser resilientes.

Metodología: El docente pone a los estudiantes en condición de reflexionar sobre estrategias formas, procedimientos para acercarse a amigos, compañeros y vecinos para reflexionar sobre las drogas sus efectos y cómo en unos casos evitar caer en este flagelo y en otros superar esta problemática. Al finalizar el estudiante evalúa en la estrategia propuesta lo positivo, negativo e interesante, mediante la plataforma virtual dentro de una semana de trabajo, desde cada uno de sus hogares.

Temporalización: Se realiza en dos semanas.

Fase Inicial

El docente utiliza la técnica Brain writing, para dar la posibilidad de elección de convertirse en promotor de salud entre sus compañeros. Consiste en recopilar ideas de los estudiantes a través de una tabla de 4 columnas y tantas filas como personas haya en el grupo. Se escriben 5 ideas de cómo hacer de promotor par, mientras que va pasando por los participantes y se van agregando más ideas y perfeccionando las que están hasta completar una estrategia mucho más potente que las aportaciones individuales.

Fase Media

Se analizarán en la estrategia global construida lo positivo, negativo e interesante para su desarrollo y ejecución, así como los avances que se piensan obtener, todo a través de la plataforma.

\section{Fase Final}

Se efectuará la conclusión de la intervención formativa por parte de los tutores, a partir del Modelo de Instrumento de Evaluación en la participación, con el objetivo de valorar el impacto en los factores determinantes de resiliencia: Comunicación clara y abierta entre los miembros del sistema familiar; expresión franca de emociones y sentimientos; solidaridad y colaboración en el enfrentamiento y la resolución de problemas; cohesión en la estructura familiar; organización familiar flexible, adecuada comunicación con el medio externo, existencia de una red de apoyo, y un sistema compartido de creencias que dote de sentido a la adversidad. 


\section{Actividad extracurricular 5: Concurso ¿Cuál es mi idea innovadora para mantenerme resiliente ante el consumo de droga?}

Objetivo: Plasmar ideas innovadoras acerca de cómo mantener la resiliencia ante el consumo de las drogas en los jóvenes.

Metodología: Interacción Social de Vygostsky.

Fase Inicial

El docente realizará el lanzamiento del concurso, sus objetivos y las bases que se tomarán en cuenta en la evaluación para seleccionar los ganadores, así como las orientaciones del proceso de inscripción.

Fase de desarrollo

Se presentan los aportes de los estudiantes de cada uno de los paralelos, las normativas bajo las cuales el jurado evaluará los trabajos y a cada uno de los que ocuparán este rol durante la competencia.

Fase final

El jurado hace la declaratoria de ganadores y premiación a partir de la evaluación del cumplimiento de las Bases del Concurso.

Temporalización: Se realiza al cierre de este ciclo de actividades.

Ubicación: en el patio de la institución.

\subsection{Evaluación de la propuesta}

Se tuvo una consulta individual con cada especialista para obtener su consentimiento vía correo electrónico para participar en la evaluación de la misma. Luego se puso a consideración de la Coordinación del Programa y la Junta Académica de la Universidad Indoamérica las hojas de vida de 4 especialistas para su aprobación y posterior valoración de la propuesta, pues es este contexto se consideran válidas las propuestas valoradas por al menos dos pares académicos que pueda hacer la función de especialistas. Ellos fueron seleccionados a partir de los criterios de formación doctorado en Educación, Pedagogía, Psicología, Promoción y Salud, publicaciones relacionadas en el ámbito escolar y curso realizados. La evaluación se realizó tomando en consideración los criterios: pertinencia dada por la relación diagnóstico de la problemática - estructura de la propuesta; viabilidad dada por la suficiencia de datos indicativa de la problemática; transferibilidad dada por la posibilidad de aplicarla en contextos con similar problemática.

Concluyen que su aplicación pudiera efectivamente denotar un cambio significativo en la concientización y prevención de los estudiantes de Educación Básica ante el consumo de drogas, fortaleciendo la resiliencia ante estos problemas, de ahí su pertinencia. Además, existe información de diferentes fuentes que evidencia la problemática por lo que es viable para el contexto donde se propone. Las autoridades y docentes tendrán una alternativa didáctica de gran valía para aplicarla en todas las unidades educativas que presenten esta problemática, pero será posible transferir solo si es comprobada la problemática diagnosticada en este estudio y además de que los directivos estén de acuerdo con su aplicación.

En sentido general, los especialistas concuerdan en la efectividad del desarrollo de la propuesta. Sin embargo, hicieron observaciones para la fase de implementación, pues esta es esencial para lograr el impacto de la propuesta y prevenir desfases e incumplimiento de los objetivos por los implicados, tales como: buscar aliados estratégicos, concientizando la problemática social para que contribuya con el reconocimiento a las buenas conductas, priorizar el orden dado en las fases y actividades, incluir otras actividades dependiendo del grado de 
aceptación de cada uno por los estudiantes, mantener una base de información continua y oportuna en cada una de las fases a partir de un registro sistemático de las distintas actividades e identificar debilidades y fortalezas de cada fase. También manifestaron que se ha tener en cuenta le metodología de diagnóstico, la complejidad de atender tal problemática en esta zona del país y el entrenamiento a docentes para su aplicación.

\section{Discusión y conclusiones}

Entre las causas que originan el consumo de drogas en la edad de la adolescencia en la literatura consultada están: la influencia del entorno en que se desarrollan los jóvenes, la carencia del apoyo familiar y desestructuración de planes futuro, la permisibilidad legal y despenalización de tenencia de pequeñas cantidades de drogas, así como el poco involucramiento de la comunidad educativa en estrategias de prevención. En el afrontamiento de esta problemática juega un papel importante la comunidad educativa que incluye a rectores, docentes, y a los propios estudiantes, en la ejecución de estrategias preventivas antes que la situación sea de mayor complejidad para su solución, puesto que la mayoría de estudiantes inician la dependencia hacia las drogas en las instituciones educativas, por problemas que no son solucionados en sus hogares.

Retomando el objetivo de diagnosticar la situación particular de una unidad educativa ecuatoriana en relación al consumo de drogas de sus estudiantes y ofrecer un sistema de actividades extracurriculares que busca la prevención del consumo y el desarrollo de actitudes de resiliencia del alumnado puede afirmarse que: los factores familiares y la no priorización de este problemas desde el punto de vista educativo resultan en un aumento de la vulnerabilidad de los estudiantes y por consiguiente a la exposición de problemas sociales y de salud cuando la tendencia es a incrementar la ingestión de ellas como lo demostró Montero et al. (2020) en su estudio.

Precisamente, dentro de los datos más destacados aportados por este estudio está el alto porcentaje de estudiantes que conocen y han consumido los diferentes tipos drogas al menos una vez, pero este dato es confuso y contradictorio con el que se refiere a que la mayoría reconoció en el consumo la sensación de bienestar y luego con el reconocimiento de la especialista DECE sobre las manifestaciones del consumo en los estudiantes. Esta evidencia es una razón por la cual debe estudiarse con mayor profundidad la relación tipo de droga que conoces, las que se consumen y la frecuencia con que lo hacen. Se requiere distinguir entre consumo de drogas y del alcohol, aunque estas pueden estar muy relacionadas por las implicaciones sociales, en el aprendizaje y para la salud en general (Cortaza et al., 2012; Costa et al., 2013; Júnior y Gaya, 2015; Loor et al., 2018; Meneses, 2020; Rial et al., 2020). Además, desde el punto de vista educativo es necesario cual fue la vía de acceso a la sustancia (TV, por algún amigo, por la escuela, la familia u otra).

También debe profundizarse en las causales, pues los problemas familiares se erigen como la principal, sin dejar de atender otros factores propios de la adolescencia como es la necesidad de socializar con otros de su edad que también consumen y lograr altos niveles de empatía, aceptación y reafirmación de identidad a través de conductas que llamen la atención de los demás. Una de ellas son las reuniones de fin de semana en el cual el consumo de alcohol y otras drogas es el generador del vínculo con los demás, tan como lo manifestó Maturana (2011).

Pese a que la institución educativa conoce la problemática, se aprecia que carece de alternativas internas e intersectoriales para hacer una intervención educativa dado su nivel de generalidad y que el ambiente hogareño no ha resultado ser aportador, todo lo contrario, la mayoría de los estudiantes refiere no tener atención de sus padres, lo cual complejiza la labor 
educativa. Ello requiere que, los profesionales de la salud y docentes, quienes tiene mejores posibilidades de acercarse a los estudiantes emprendan acciones coordinadas de promoción y autocuidado de la salud de manera integral e interdisciplinaria basado en el desarrollo de estas y otras habilidades sociales desde la escuela como lo proponen Trigozo et al. (2016) y Aciole (2016).

No obstante, al panorama que muestran los datos en este contexto específico se presentan oportunidades para la prevención a partir de la predisposición de los estudiantes de participar actividades extraescolares y fomentar la resiliencia.

La propuesta del sistema actividades extracurriculares enfocada a potenciar la resiliencia y prevenir el consumo de drogas se construyó ordenando cada actividad y diseñando varias metodologías en forma de sistema, de manera que la secuencia seguida proporcionará un aprendizaje paulatino y concientización por parte de los estudiantes que presentan la problemática. La evaluación de la propuesta por criterio de los especialistas determinó su pertinencia, viabilidad y transferibilidad para contribuir a potenciar la resiliencia y con el ello prevenir el consumo de drogas. No obstante, se sabrá cuando se aplique y considerando las condiciones sugeridas por los especialistas en cuanto al proceso de diagnóstico, la complejidad de atender tal problemática en esta zona del país y el entrenamiento a docente para que se formen y atiendan a esta problemática.

\section{Referencias}

Aciole, G. (2016). Rupturas paradigmáticas e novas interfaces entre educação e saúde. Caderno de Pesquisa, 46 (162), 1172-1191. Recuperado de https://doi.org/10.1590/198053143528.

Amar, J., Kotliarenko, M., y Abello, R. (2003). Factores psicosociales asociados con la resiliencia en niños colombianos víctimas de violencia intrafamiliar. Investigación y Desarrollo, 11(1), 18-35.

Becoña, E. (2006). Resiliencia y consumo de drogas: una revisión. Adicciones, 15(1), 67-78.

Cajizo, H. (1975). Teoría General del Sistema curso de Administración Pública. San José, Costa Rica.

Chiavenato, I. (2017). Administración de Recursos Humanos. Recuperado de https://cucjonline.com/biblioteca/files/original/aec4d0f8da9f45c14d9687966f292cd2.pdf.

CONSEP. (2005). Segunda Encuesta Nacional a estudiantes a de educación media sobre consumo de drogas. Quito, Ecuador: CONSEP.

Cortaza, L., Villar, M.A., y Puig, A. (2012). Consumo de drogas en adolescentes mexicanas. Rev. Iberoam. Educ. Investi. Enferm, 2(3), 19-25. Recuperado de https://doi.org/10.1590/ S0104-11692004000700008.

Cortez, J. (2010). Resilience: A View from Nursing. Ciencia y Enfermería, 16(3), 27-32.

Costa, E. M., de Araújo, L. L., da Silva, N. M., y Luck, I. C. (2013). Teatro como recurso terapêutico na prevenção ao uso de drogas: percepção de adolescentes. Revista Brasileira Em Promoção Da Saúde, 26(3), 333-339. Recuperado de https://doi.org/10.5020/18061230.2013. p333.

García, C. (2015). Concepto de vulnerabilidad psicosocial en el ámbito de la salud y las adicciones. Health and Addictions, 15(1), 1-10.

Júnior, G.A., y Gaya, C.M. (2015). Implicações do uso de álcool, tabaco e outras drogas na vida do universitário. Revista Brasileira Em Promoção Da Saúde, 28(1), 67-74. Recuperado de https://periodicos.unifor.br/RBPS/article/view/3166. 
Loor, W., Hidalgo, H., Macías, J., García, E., y Scrich, A. (2018). Causas de las adicciones en adolescentes y jóvenes en Ecuador. Archivo Médico de Camagüey, 22(2), 1-5.

Maturana, A. (2011). Consumo de alcohol y drogas en adolescentes. Revista de Medicina Clínica, 22 (1), 98-109. Recuperado de https://doi.org/10.1016/S0716-8640(11)70397-2.

Meneses, C. (2020). La triada adictiva: consumo de cocaína, alcohol y sexo. Revista Española de Drogodependencia, 45(1), 26-35.

Montero, P., Reyes, M. F., Cardozo, F., Brown, E. C., Pérez, A., Mejía, J., Aguilar, J., y Paredes, M. (2020). Adolescent substance use and its association with risk and protective factors. An exploratory analysis of the large-scale school survey of Colombia. Adicciones, 32(2), 105-115. Recuperado de https://doi.org/10.20882/adicciones.1083.

Moraes, M., Pinto, F., Pereiras, C., Agustín, C., Duarte, V., Barceló, J., Sosa, C., y González, G. (2020). Autodeclaración del consumo de marihuana, tabaco, alcohol y derivados de cocaína en embarazadas en 2013 y 2016, Montevideo, Uruguay. Adicciones, 32(3), 173-180.

OMS. (1994). Glosario de términos de Alcohol y drogas. Recuperado de https://www.who.int/ substance_abuse/terminology/lexicon_alcohol_drugs_spanish.pdf.

ONU. (2010). Informe Mundial sobre las Drogas 2010. París, Francia, ONU.

Pavón, L. (2020). Sobre práctica de ocio juvenil y consumos de drogas en áreas rurales con perspectiva de género. Revista Española de Drogodependencia, 45(1), 69-85.

Plazas, N. (2020). La ONU advierte de los efectos de pandemia en el tráfico mundial de drogas. France24. Recuperado de https://www.france24.com/es/20200626-naciones-unidas-trafico-drogas-pandemia.

Rial, A., Golpe, S., Barreiro, C., Gómez, P., y Isorna, M. (2020). The age of onset for alcohol consumption among adolescents: Implications and related variables. Adicciones, 32(1), $52-62$.

Riofrío, R., y Nascimento, L. C. (2010). Consumo de drogas en los jóvenes de la ciudad de Guayaquil, Ecuador. Revista Latino-Americana de Enfermagem, 18(spe), 598-605. Recuperado de https://dx.doi.org/10.1590/S0104-11692010000700016.

Rivadeneira, M., Sola, H., Chuquimarca, C., Ocaña A., Navas, J., León, G.A., Dávila, S.M., Villalba, J.J., y Cóndor D.J. (2020). Experiencia y resultados de un proceso educativo interdisciplinario para la promoción de salud en universitarios. Hacia. Promoc. Salud., 25(2), 109-124. Recuperado de https://doi.org/10.17151/hpsal.2020.25.2.12.

Rivero, Y., Suárez, N., y Fernández, E. V. (2017). Promoción y educación para la salud en el contexto universitario ecuatoriano: desafíos y propuesta de desarrollo profesional. Revista Conrado, 14(61), 125- 132. Recuperado de http://conrado.ucf.edu.cu/index.php/conrado.

Romo, N. (2020). Propuestas sobre género y masculinidades en el estudio de los usos y abusos de drogas. Revista Española de Drogodependencia, 45(1), 5-9.

Sánchez, A. M., y García, A. (2008). Prevención del abuso de drogas y promoción de la salud en los jóvenes. REXE. Revista de Estudios y Experiencias en Educación, (14), 117-130.

Trigozo, M. G., Alvan, C.I., y Santillan, K.A. (2016). Factores familiares asociados al consumo de drogas psicoactivas, en estudiantes de secundaria de la institución educativa Simón Bolivar, Iquitos 2016. (Tesis de Grado). Universidad Nacional de la Amazonía Peruana, Perú.

UNESCO. (2018). Respuestas del sector de la educación frente al consumo de alcohol, tabaco y drogas. UNESCO Biblioteca Digital. Recuperado de https://unesdoc.unesco.org/ ark:/48223/pf0000262942.

Villarreal, J. A. (2020). Sinergia educación, prevención, promoción de salud comunitaria desde el paradigma de la sociocultura. Hacia. Promoc. Salud., 25(2), 39-53. Recuperado de https://doi.org/10.17151/hpsal.2020.25.2.7. 